\title{
DIFFERENTIAL QUADRATURE METHOD FOR VIBRATION ANALYSIS OF PRESTRESSED BEAMS
}

\author{
Li Peng ${ }^{1}$, Ying Wang ${ }^{1}$ \\ ${ }^{1}$ College of Civil Engineering, Shanghai Normal University, Shanghai 201418, China
}

\begin{abstract}
This paper investigates natural frequencies of free transverse vibrations of prestressed beams, and the governing equations and natural frequencies of the free vibration in related literatures are discussed and corrected. The differential quadrature methods (DQ) are applied directly to the corrected governing equations to get the the values of natural frequency numerically. Under the simple supported boundary conditions, the natural frequencies of model beam are numerically studied, and the physical parameters of the beam are analyzed respectively. The numerical results show that the natural frequency values increase with the growth of concrete strength and eccentricity of prestressed steels. But with the increase of the span length of beam and values of original prestressing force, the natural frequency values decrease.
\end{abstract}

\section{Introduction}

Prestressed concrete technique can effectively improve the cracking of concrete structure, improve the stiffness and durability of the structure, and give full play to the high strength performance of materials, which has been widely used in civil engineering ${ }^{[1-3]}$. In recent years, many researchers have studied the static performance of prestressed concrete structure, but the investigations on the complex dynamic performance are relatively insufficient.

The differential quadrature (DQ) method is a powerful tool for dealing with dynamical problems. Firstly it was introduced for structural analysis by Bert et al. ${ }^{[4]}$. and since then it has been successfully employed for the analysis of vibration of beams ${ }^{[5-6]}$.

For the vibration characteristics of prestressed beams, the formulas of different linear vibration frequencies are derived by applying the theory of material mechanics and structural dynamics ${ }^{[3,7]}$, However, these formulas are based on simplified vibration control equations, and their accuracy is worth discussing. In this paper, the existing vibration control equations of prestressed beams are analyzed and corrected, and the differential quadrature method (DQ) is directly applied to the corrected equations. The differential equations are transformed to a generalize $\mathrm{d}$ eigenvalue problem, and the numerical solutions of natural frequency are obtained based on the method of DQ. Under the simply supported boundary conditions, the first four order numerical solutions of natural frequency of model beam are given, and the different effects of physical parameters of prestressed beam and prestressed steel on natural frequency are analyzed.
The present paper is organized as follows. Section 2 establishes the governing equation for the transverse free vibration of a finite elastic prestressed beam. Section 3 develops the DQ schemes to descretize the governing equation. Section 4 presents numerical examples for investigations on the effects of the different parameters of the relevant beam and prestressed steel on the natural frequencies. Section 5 ends the paper with the concluding remarks.

\section{Equation of motion}

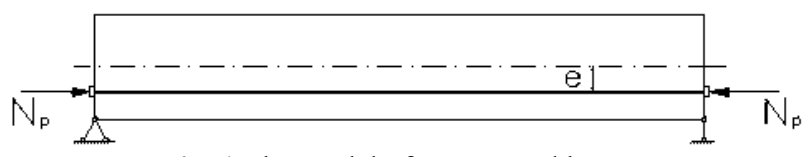

Fig. 1 The model of prestressed beam

Free transverse vibration of a prestressed beam can be described by transverse deflection of the cross section due to bending moment, depending on axial coordinate $x$ and time $t$.

Fig. 1 shows a finite elastic prestressed concrete beam with a length $l$, the bending moment and the axial force can be expressed as

$$
\left\{\begin{array}{l}
N_{p}=N_{P^{0}}+\Delta N_{p} \\
M=N_{p} e=\left(N_{p^{0}}+\Delta N_{p}\right) e
\end{array}\right.
$$

The free vibration equation of a prestressed beam is governed by the equation ${ }^{[1]}$

$$
\frac{\partial^{2}}{\partial x^{2}}\left(E I \frac{\partial^{2} y}{\partial x^{2}}\right)+\frac{\partial^{2}}{\partial x^{2}}\left(N_{P} y\right)-\frac{\partial^{2}}{\partial x^{2}}(M)+m \frac{\partial^{2} y}{\partial t^{2}}=0
$$

Substituting Eq. (1) into Eq. (2) yields

$$
\frac{\partial^{2}}{\partial x^{2}}\left(E I \frac{\partial^{2} y}{\partial x^{2}}\right)+\frac{\partial^{2}}{\partial x^{2}}\left[\left(N_{P^{0}}+\Delta N_{p}\right) y\right]-\frac{\partial^{2}}{\partial x^{2}}\left[\left(N_{P^{0}}+\Delta N_{p}\right) e\right]+m \frac{\partial^{2} y}{\partial t^{2}}=0
$$


(3)

where $y, m$ and $E I$ are vibration displacement, unit mass, and bending flexural rigidity of the beam. $e, N_{p 0}$ and $\Delta N_{P}$ are eccentricity, initial prestressing force and increased prestressing force due to flexural vibration.

It is generally accepted that $y$ is much less than $e$, so the value of $\Delta N_{P} y$ is negligibly small, and Eq.(3) can be rewritten as

$$
E I \frac{\partial^{4} y}{\partial x^{4}}+\frac{\partial^{2}}{\partial x^{2}}\left(N_{P^{0}} y\right)-e \frac{\partial^{2}}{\partial x^{2}}\left(\Delta N_{P}\right)+m \frac{\partial^{2} y}{\partial t^{2}}=0
$$

Based on the assumption that increased prestressing forces $\Delta N_{P}$ is proportional to midspan deflection $y$, the horizontal displacement due to the applied midspan load $F$ can be calculated from the equation

$$
\delta=\int \frac{m_{1} M}{E I} d x=\frac{F l^{2} e}{8 E I}
$$

The horizontal displacement caused by the unit force acting on the anchorage point can be obtained as

$$
\delta_{1}=\int \frac{m_{1}^{2}}{E I} d x+\int \frac{N_{1}^{2}}{E A} d x=\frac{l e^{2}}{E I}+\frac{l}{E A}+\frac{l_{t}}{E_{t} A_{t}}
$$

The change of anchoring force $\Delta N_{P}$ caused by load $F$ is

$$
\Delta N_{P}=\frac{\delta}{\delta_{1}}=\frac{\int \frac{m_{1} M}{E I} d x}{\int \frac{m_{1}^{2}}{E I} d x+\int \frac{N_{1}^{2}}{E A} d x}=\frac{\frac{F l^{2} e}{8 E I}}{\frac{l e^{2}}{E I}+\frac{l}{E A}+\frac{l_{t}}{E_{t} A_{t}}}=\frac{F l e}{8\left(e^{2}+\lambda\right)}
$$

where $\lambda=\frac{I}{A}+\frac{E I}{E_{t} A_{t}} \frac{l_{t}}{l}, A, I, l$ and $E I$ are

cross-sectional area, cross-sectional moment of inertia, span length and bending flexural rigidity of the beam. $l_{t}$ and $E_{t} A_{t}$ are length and flexural rigidity of prestressed reinforcements.

From Eq. (7), the load $F$ can be given in the form

$$
F=\frac{8\left(e^{2}+\lambda\right)}{l e} \Delta N_{p}
$$

The deflection of the midpoint of a beam under the action of force $F$ is

$$
y_{F}=\frac{l^{3}}{48 E I} F
$$

Substituting Eq. (8) into Eq. (9) yields

$$
y_{F}=\frac{l^{2}\left(e^{2}+\lambda\right)}{6 E I e} \Delta N_{p}
$$

By the displacement reciprocal theorem, the upward displacement at midpoint due to $\Delta N_{P}$ is

$$
y_{\Delta N_{p}}=\frac{l^{2} e}{8 E I} \Delta N_{p}
$$

Deflection $y$ can be calculated as

$$
y=y_{F}-y_{\Delta N_{p}}=\frac{l^{2}\left(e^{2}+\lambda\right) \Delta N_{P}}{6 E I e}-\frac{l^{2} e \Delta N_{P}}{8 E I}=\frac{l^{2}\left(e^{2}+4 \lambda\right)}{24 E I e} \Delta N_{p}
$$

Therefore increased prestressing forces $\Delta N_{P}$ can be expressed as

$$
\Delta N_{p}=\frac{24 E I e}{l^{2}\left(e^{2}+4 \lambda\right)} y
$$

Substitution of Eq. (13) into Eq. (4) yields

$$
E I \frac{\partial^{4} y}{\partial x^{4}}+\left[N_{P^{0}}-\frac{24 E I e^{2}}{l^{2}\left(e^{2}+4 \lambda\right)}\right] \frac{\partial^{2} y}{\partial x^{2}}+m \frac{\partial^{2} y}{\partial t^{2}}=0
$$

Eq. (14) is the free vibration equation of prestressed beam with straight prestressed steel. It is derived by applying the theory of material mechanics and structural dynamics.

With method of separation of variables, the vibration solution to Eq. (14) can be assumed as

$$
y=X_{(x)} \cdot e^{i \omega_{n} t}
$$

Substituting Eq. (15) into Eq. (14) yields

$$
E I \frac{d^{4} X}{d x^{4}}-\left[N_{P^{0}}-\frac{24 E I e^{2}}{l^{2}\left(e^{2}+4 \lambda\right)}\right] \frac{d^{2} X}{d x^{2}}=m \omega^{2} X
$$

For a simply supported beam, $X(x)$ can be expressed as

$$
X(x)=A \sin \frac{n \pi}{L} x,(n=1,2,3,4 \mathrm{~L})
$$

Substitution of Eq. (17) into Eq. (16) yields

$$
E I\left(\frac{n \pi}{L}\right)^{4}+\left[N_{P^{0}}-\frac{24 E I e^{2}}{L^{2}\left(e^{2}+4 \lambda\right)}\right]\left(\frac{n \pi}{L}\right)^{2}=m \omega^{2}
$$

Solving Eq. (18), the explicit formulae for natural circular frequencies can be obtained

$$
\omega_{n}=\sqrt{\frac{E I}{m}} \cdot\left(\frac{n \pi}{l}\right)^{2} \xi
$$

where

$$
\zeta=\sqrt{1-\left(\frac{l}{n \pi}\right)^{2} \frac{N_{P^{0}}}{E I}+\frac{24}{(n \pi)^{2}} \frac{e^{2}}{\left(e^{2}+4 \lambda\right)}}
$$

With Eq. (19), the natural circular frequencies of free vibration of a prestressed beam can be easily calculated. But it must be noticed that these formulas are based on simplified vibration control equations, and their accuracy is worth discussing.

$$
\begin{aligned}
& \text { In paper [2], Eq. (14) is rewritten as }{ }^{[2]} \\
& E I \frac{\partial^{4} y}{\partial x^{4}}+m \frac{\partial^{2} y}{\partial t^{2}}+\left[N_{P^{0}}-\frac{6 E I e^{2}}{\left(e^{2}+4 \lambda\right)\left(l x-x^{2}\right)}\right] \frac{\partial^{2} y}{\partial x^{2}} \\
& +\frac{12 E I e^{2}(l-2 x)}{\left(e^{2}+4 \lambda\right)\left(l x-x^{2}\right)^{2}} \frac{\partial y}{\partial x}-\frac{12 E I e^{2}\left(3 x^{2}-3 l x+l^{2}\right)}{\left(e^{2}+4 \lambda\right)\left(l x-x^{2}\right)^{3}} y=0
\end{aligned}
$$

It is a complex partial differential equation with variable coefficients, which cannot be directly solved to obtain the analytical expression of natural frequency.

In paper [3], considering $\Delta N_{P}$ is proportional to the midpoint $(x=l / 2)$ deflection of the beam, the simplified equation is given by

$$
E I \frac{\partial^{4} y}{\partial x^{4}}+\left[N_{P^{0}}-\frac{24 E I e^{2}}{l^{2}\left(e^{2}+4 \lambda\right)}\right] \frac{\partial^{2} y}{\partial x^{2}}+m \frac{\partial^{2} y}{\partial t^{2}}=0
$$

Solving the equation with method of separation of variables, the explicit formulae for natural circular frequencies can be obtained ${ }^{[3]}$. Comparing two equations, it can be found that the last two items of Eq. (21) disappear in Eq. (22). However, when $x=l / 2$ is substituted into Eq. (21), the coefficient before y term is not zero, 
and this term mainly reflects the influence of eccentricity on vibration performance. So the accuracy of the analytical expression of natural frequency obtained by Eq. (22) is also worth discussing.

Considering the coefficient before y term of Eq. (21) is not zero, this paper corrected the Eq. (22) as

$$
E I \frac{\partial^{4} y}{\partial x^{4}}+\left[N_{P^{0}}-\frac{24 E I e^{2}}{l^{2}\left(e^{2}+4 \lambda\right)}\right] \frac{\partial^{2} y}{\partial x^{2}}-\frac{192 E I e^{2}}{l^{4}\left(e^{2}+4 \lambda\right)} y+m \frac{\partial^{2} y}{\partial t^{2}}=0
$$

To simplify the expression, the Eq. (23) can be rewritten as

$$
\rho A y,_{t t}+E I y,_{x x x x}+k_{P} y,_{x x}-k_{e} y=0
$$

where

$$
k_{P}=N_{P^{0}}-\frac{24 E I e^{2}}{l^{2}\left(e^{2}+4 \lambda\right)}, k_{e}=\frac{192 E^{2} e^{2}}{l^{4}\left(e^{2}+4 \lambda\right)}
$$

Introducing the non-dimensional quantities into Eq. (24) results in the non-dimensional form

$$
y,_{t t}+k_{\mathrm{f}}{ }^{2} y,_{x x x x}+k_{1} y,_{x x}-k_{2} y=0
$$

For simply supported beams, the dimensionless boundary conditions can be expressed by

$$
y(0, t)=y(1, t)=0, \quad y^{\prime \prime}(0, t)=y^{\prime \prime}(1, t)=0
$$

\section{Differential quadrature method}

Eq.(26) is a complex partial differential equation, and the analytical expression of natural frequency cannot be obtained directly. In here, the method of DQ is employed to numerically solve the problem. This method requires to descretize the domain of the problem into $N$ points. Then the derivatives at any point are approximated by a weighted linear summation of all the functional values along the descretized domain.

Consider the computational domain $0 \leq x \leq 1$ of the beam. The number of sampling point is $N$ in the $x$ direction. Introduce $N$ sampling point as ${ }^{[5]}$

$$
\begin{aligned}
& x_{1}=0, x_{N}=1, \\
& x_{i}=\frac{1}{2}\left[1-\cos \left(\frac{i-1}{N-1} \pi\right)\right](i=2,3, \cdots, N-1)
\end{aligned}
$$

The quadrature rules for the derivatives of a function at the sampling points yield

$$
\begin{aligned}
& y,_{x x}\left(x_{i}, t\right)=\sum_{j=1}^{N} A_{i j}^{(2)} y\left(x_{j}, t\right), \\
& y,_{x x x x}\left(x_{i}, t\right)=\sum_{j=1}^{N} A_{i j}^{(4)} y\left(x_{j}, t\right)
\end{aligned}
$$

Substitution of Eq. (29) into Eq. (26) yields

$$
\ddot{y}_{i}+\sum_{k=1}^{N}\left(k_{\mathrm{f}}^{2} A_{i k}^{(4)}+k_{1} A_{i k}^{(2)}\right) y_{k}-k_{2} y_{i}=0
$$

where

$$
y_{i}(t)=y\left(x_{i}, t\right)
$$

under simply supported boundary conditions

$$
y_{1}=y_{N}=0, \sum_{k=1}^{N} A_{1 k}^{(2)} y_{k}=\sum_{k=1}^{N} A_{N k}^{(2)} y_{k}=0
$$

where the weight coefficient is defined by

$$
A_{i k}^{(1)}=\left\{\begin{array}{l}
\prod_{\mu=1, \mu \neq i}^{N}\left(x_{i}-x_{\mu}\right) /\left[\left(x_{i}-x_{k}\right) \prod_{\mu=1, \mu \neq k}^{N}\left(x_{k}-x_{\mu}\right)\right] \\
(i, k=1,2, \cdots, N, \quad i \neq k) \\
\sum_{\mu=1, \mu \neq i}^{N} \frac{1}{x_{i}-x_{\mu}} \quad(i=1,2, \cdots, N, i=k)
\end{array}\right.
$$

and in case of $r=2,3, \ldots, N-1$

$$
A_{i k}^{(r)}=\left\{\begin{array}{lr}
r\left(A_{i i}^{(r-1)} A_{i k}^{(1)}-\frac{A_{i k}^{(r-1)}}{x_{i}-x_{k}}\right) & (i, k=1,2, \cdots, N, i \neq k) \\
-\sum_{\mu=1, \mu \neq i}^{N} A_{i \mu}^{(r)} & (i=1,2, \cdots, N, i=k)
\end{array}\right.
$$

To overcome difficulties in the implementation of the boundary conditions, the modified weighting coefficient matrix are introduced as

$$
\begin{gathered}
{\left[\tilde{A}^{(2)}\right]=\left[\begin{array}{cccc}
0 & 0 & \cdots & 0 \\
A_{21}^{(2)} & A_{22}^{(2)} & \cdots & A_{2 N}^{(2)} \\
\vdots & \vdots & \ddots & \vdots \\
A_{(N-1) 1}^{(2)} & A_{(N-1) 2}^{(2)} & \cdots & A_{(N-1) N}^{(2)} \\
0 & 0 & \cdots & 0
\end{array}\right]} \\
{\left[\tilde{A}^{(r)}\right]=\left[A^{(1)}\right]\left[\tilde{A}^{(r-1)}\right] \quad(r=3,4, \cdots)}
\end{gathered}
$$

Substituting matrix (35) and (36) into Eq. (30), one obtain

$$
\ddot{y}_{i}+\sum_{k=1}^{N}\left(k_{\mathrm{f}}^{2} \tilde{A}_{i k}^{(4)}+k_{1} \tilde{A}_{i k}^{(2)}\right) y_{k}-k_{2} y_{i}=0
$$

Eq. (37) leads to a generalized eigenvalue problem that can be numerically solved.

\section{Numerical results}

Consider a finite elastic prestressed concrete model beam, the parameters of the beam are listed in Table 1. Under pinned boundary conditions, from Eq. (37), the values of natural frequency can be found by numerical method.

Table 1 Properties of the beam

\begin{tabular}{l|l|l}
\hline Item & Notation & Value \\
\hline Young's modulus of concrete & $E_{c}$ & $31.5 \mathrm{GPa}$ \\
\hline Young's modulus of prestressed steel & $E_{t}$ & $195 \mathrm{GPa}$ \\
\hline Mass density & $\rho$ & $2.6 \times 10^{3} \mathrm{~kg} / \mathrm{m}^{3}$ \\
\hline Cross sectional area & $A$ & $8 \times 10^{4} \mathrm{~mm}^{2}$ \\
\hline Second moment of area & $I$ & $2.67 \times 10^{8} \mathrm{~mm}^{4}$ \\
\hline Eccentricity & $e_{0}$ & $30 \mathrm{~mm}$ \\
\hline Length & $L$ & $7 \mathrm{~m}$ \\
\hline
\end{tabular}

For the free motion of the model beam, the effects of parameters on the natural frequencies are respectively studied. In Table 2, the first four natural frequencies of the model beam with various concrete strength are given. For same concrete strength, with the growth of the orders, the natural frequencies increase greatly, but for the beam with higher concrete strength, the natural frequencies increase slightly. In Table 3, it shows that for same span length, with the growth of the orders, the natural frequencies increase significantly, but with the increase of span length, the natural frequencies decrease a little, 
and the natural frequency values of higher orders decrease more quickly.

Table 2 Natural frequencies with various concrete strength

\begin{tabular}{c|cccc}
\hline & $f_{1}$ & $f_{2}$ & $f_{3}$ & $f_{4}$ \\
\hline C20 & 19.381 & 74.476 & 166.271 & 294.780 \\
C30 & 20.700 & 79.988 & 178.778 & 317.080 \\
C35 & 21.085 & 81.602 & 182.441 & 323.612 \\
C40 & 21.308 & 82.542 & 184.577 & 327.423 \\
\hline
\end{tabular}

Table 3 Natural frequencies with various beam length

\begin{tabular}{c|cccc}
\hline$L$ & $f_{1}$ & $f_{2}$ & $f_{3}$ & $f_{4}$ \\
\hline 6.5 & 24.335 & 94.517 & 211.465 & 375.190 \\
7.0 & 21.085 & 81.602 & 182.441 & 323.612 \\
7.5 & 18.461 & 71.182 & 159.024 & 282.000 \\
8.0 & 16.312 & 62.652 & 139.858 & 247.942 \\
\hline
\end{tabular}

Table 4 Natural frequencies with various original prestressing force

\begin{tabular}{c|cccc}
\hline$N_{\mathrm{P} 0}$ & $f_{1}$ & $f_{2}$ & $f_{3}$ & $f_{4}$ \\
\hline 600 & 21.394 & 81.923 & 182.764 & 323.936 \\
800 & 21.240 & 81.763 & 182.603 & 323.774 \\
1000 & 21.085 & 81.602 & 182.441 & 323.612 \\
1200 & 20.929 & 81.441 & 182.279 & 323.450 \\
\hline
\end{tabular}

Table 5 Natural frequencies with various eccentricity

\begin{tabular}{c|cccc}
\hline$e_{0}$ & $f_{1}$ & $f_{2}$ & $f_{3}$ & $f_{4}$ \\
\hline 20 & 20.976 & 81.490 & 182.328 & 323.499 \\
30 & 21.085 & 81.602 & 182.441 & 323.612 \\
50 & 21.326 & 81.889 & 182.793 & 324.051 \\
80 & 21.684 & 82.265 & 183.171 & 324.432 \\
\hline
\end{tabular}

In Table 4 and Table 5, with the increase of the values of the original prestressing force, the natural frequencies decrease slightly, but with the growth of eccentricity, the natural frequencies increase a little.

\section{Conclusions}

This paper investigates natural frequencies of free transverse vibrations of a finite prestressed beams under simply supported boundary conditions, and the free vibration governing equations in related literatures are discussed and recorrected. As for the complex partial differential equation, the analytical expression of natural frequency cannot be obtained directly, the differential quadrature methods are applied to get the numerical results, and the effects of the physical parameters of the beam on the natural frequencies are respectively analyzed. The numerical results show that the natural frequency values increase with the growth of concrete strength and eccentricity of prestressed steels. But with the increase of the beam span length and values of original prestressing force, the natural frequency values decrease.

\section{References}

1. Ayaho M., Katsuji Tei. Behavior of Prestressed Beam Strengthened with External Tendons. J. Struct Eng 9, ASCE(2000).

2. Menglin L., Tingting H. Mode perturbation method for lateral vibration analysis of prestressed beams. J. Prestress Technology 1, (2010).

3. Xueyu X., Shousheng W. Analysis and research of externally prestressed concrete beam vibration behavior. J. Earthq Eng Eng Vib 2, (2005).

4. Bert, C.W., Malik, M. Differential quadrature method in computational mechanics: A review. Appl Mech Rev., 49, (1996).

5. Malekzadeh P., Karami G. DQEM for free vibration analysis of Timoshenko beams on elastic foundations. Comput Mech 31, (2003).

6. Magrab E.B. Natural Frequencies and Mode Shapes of Timoshenko beams with attachmens. J. Vib Control 13, 7, (2007).

7. Li P. Analysis of natural frequency of externally prestressed concrete beams with asymmetric configuration. J. Shanghai Normal University (Natural Sciences) 40, 5, (2011). 